\title{
Knowledge of chronic total occlusion among Polish interventional cardiologists
}

\author{
Krzysztof L. Bryniarski ${ }^{1}$, Michał Zabojszcz ${ }^{2}$, Grzegorz Dębski ${ }^{2}$, Jakub Marchewka², Jacek Legutko³, \\ Sławomir Surowiec ${ }^{4}$, Zbigniew Siudak ${ }^{1}$, Krzysztof Żmudka' ${ }^{1}$, Dariusz Dudek ${ }^{3}$, Leszek Bryniarski ${ }^{4}$ \\ ${ }^{1}$ Department of Interventional Cardiology, Cardiology Institute, Jagiellonian University Medical College, John Paul II Hospital, Krakow, \\ Poland \\ 2Department of Cardiology, Dietl's Hospital, Krakow, Poland \\ 3I Department of Cardiology and Cardiovascular Interventions, Cardiology Institute, Jagiellonian University Medical College, \\ University Hospital, Krakow, Poland \\ ${ }^{4}$ I Department of Cardiology Interventional Electrocardiology and Arterial Hypertension, Cardiology Institute, Jagiellonian University \\ Medical College, University Hospital, Krakow, Poland
}

Postep Kardiol Inter 2015; 11, 2 (40): 89-94

DOI: $10.5114 /$ pwki.2015.52280

\begin{abstract}
A bstract
Introduction: Chronic total occlusion (CTO) recanalization is indicated in patients with symptoms and evidence of ischemia, but in most cases those types of lesions are still treated medically. In the last few years CTO angioplasty technique has changed dramatically due to considerable advances in techniques and dedicated equipment.

Aim: An attempt to assess the state of knowledge of technical aspects of CTO angioplasty of coronary arteries among Polish interventional cardiologists.

Material and methods: Questionnaire survey performed during two major Polish invasive cardiology workshops.

Results: In the study there participated 113 physicians with an average length of work experience of 13 years, most of them cardiologists certified as independent primary operators. The majority of respondents recognized the need of prevention of thrombotic complications through control of activated coagulation time during the CTO procedures. Prevention of renal complications and X-ray protection are also recognized as a significant part of the procedures. The benefits from the use of over-the-wire microcatheters and balloons, the proper choice of dedicated guidewires, contralateral injections and retrograde technique are underestimated.

Conclusions: Despite satisfactory knowledge about indications and qualification for the CTO procedure, the awareness of procedural aspects (particularly the retrograde technique) as well as the dedicated CTO equipment among Polish interventional cardiologists is still insufficient.
\end{abstract}

Key words: percutaneous coronary intervention, chronic total occlusion, physicians' knowledge.

\section{Introduction}

Chronic total occlusion (CTO) of the coronary artery is currently defined as the presence of thrombolysis in myocardial infarction (TIMI) 0 flow within the occluded segment, and the estimated duration is over 3 months [1]. The reported prevalence of CTOs is generally about $20 \%$ in large registries [2]. The CTO recanalization is indicated in patients with symptoms and evidence of ischemia, but those types of lesions are in most cases treated medically based on the misconception that well-developed collateral vessels are sufficient to supply the myocardium in the area of occluded arteries [3-7].
The CTO angioplasty technique has changed dramatically in the last years due to considerable advances in techniques (both antegrade and retrograde) and dedicated equipment (wires, microcatheters and other CTO dedicated devices). Nowadays procedures are even more technically demanding, time consuming and require specific knowledge. Also meticulous procedural planning, $X$-ray protection and prevention of contrast-induced nephropathy are of paramount importance.

In specialised centres using the modern devices and new techniques the efficacy of revascularization procedures of CTO has improved and reached up to $90 \%$ even when attempting more complex СTO than 10 years ago

\section{Corresponding author:}

Krzysztof L. Bryniarski, Dietl's Hospital, 17 Kopernika St, 31-501 Krakow, Poland, phone: +48 1242473 00, e-mail: I_bryniarski@poczta.fm Received: 27.08.2014, accepted: 28.08.2014. 
[8]. Those findings are in concordance with our own data: in the last 3 years we achieved an $88.2 \%$ success rate [9]. The CTO angioplasty is a highly individualized discipline, but there are some general principles and consensuses. According to unpublished data of the Association of Cardiovascular Interventions of the Polish Cardiac Society (ACVI), during the first 6 months of 2014 in Poland 601 CTO procedures were performed, with a $54 \%$ success rate $(50 \%$ patients with TIMI 3 flow after the procedure and $4 \%$ with TIMI 2). This efficacy is less than the $77 \%$ reported in Patel's meta-analysis, but similar to previous Polish publications [10, 11].

As we described previously in 2010, the level of knowledge regarding СTO of coronary arteries was not sufficient [6]. Since then the new consensus of the EuroCTO Club has been published, and the literature of this subject is very large; for example, in 2013 more than 70 important articles were published [12].

\section{Aim}

The aim of our study was to evaluate knowledge about technical aspects of percutaneous CTO recanalization among Polish interventional cardiologists.

\section{Material and methods}

Our study was conducted among cardiologists and physicians interested in the topic of CTO. For that a self-written questionnaire in Polish was used. The majority of the questions were closed questions with 2 to 6 answers to choose, most with multiple choices. The first questions characterised the participants of the study, the next ones concerned knowledge about CTO, and those results were published in a separate paper.

The study was conducted during the $14^{\text {th }}$ workshops of New Frontiers in Interventional Cardiology (NFIC) held

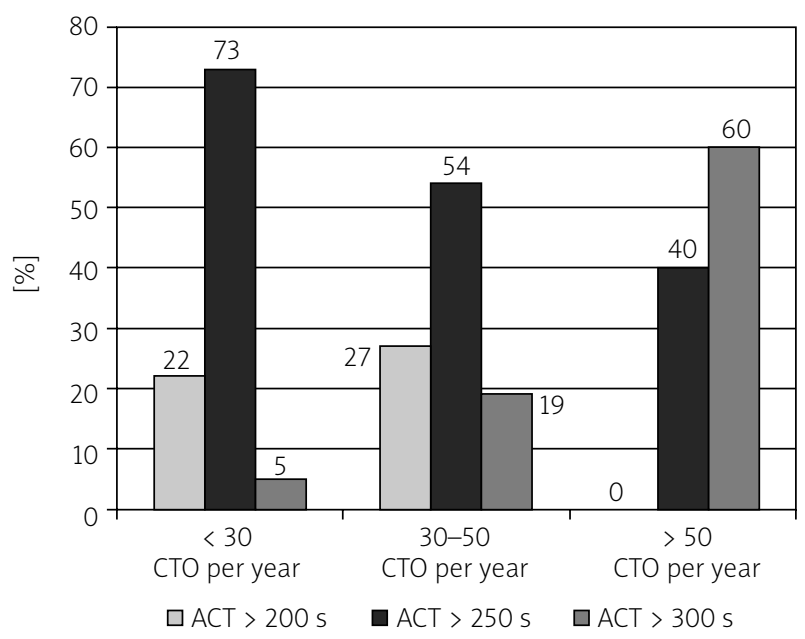

Figure 1. Preferred target ACT level during recanalization according to declared annual volume of $\mathrm{PCl}$ in $\mathrm{CTO}$ in Krakow on 27-30 November 2013 and at the $18^{\text {th }}$ Warsaw Course on Cardiovascular Interventions (WCCI) which was held on 9-11 April 2014.

\section{Statistical analysis}

The comparison of numbers and percentages of cases with regards to the whole analyzed group is presented. To assess the differences in the nominal variables, non-parametric chi-squared $\left(\chi^{2}\right)$ Pearson's test, Yates' $\chi^{2}$ test or Fisher's exact test was applied (depending on the number of subjects in subgroups). In order to assess the strength of the relations between variables, Spearman's correlation test was used. Results with a $p$ value of $<0.05$ were considered significant. Statistical analysis was carried out using Statistica 8 PL software (StatSoft Inc.).

\section{Results}

113 physicians completed the questionnaire in our study. The average length of work experience was 13 years (34\% worked over 15 years). Most of the respondents had a medical doctor title (59\%) and had completed or were in the course of the specialization in cardiology (58\% and 33\% respectively). The majority of the physicians were employed in a non-academic cardiology ward with a catheterization laboratory $(71 \%)$ or in an academic cardiology clinic with a catheterization laboratory (27\%). $73 \%$ of respondents identified themselves as independent operators. The declared number of diagnostic coronary angiographies performed during the last year was 340 on average, and the declared number of percutaneous coronary interventions was 177 on average (including an average of 23 CTO procedures per year). The estimated success rate of percutaneous coronary intervention $(\mathrm{PCI})$ in $\mathrm{CTO}$ procedures in the whole studied group was $63.5 \%$. Detailed characteristics of the studied group are presented in another article [13].

The majority of respondents (86.5\%) recognized the need of routine assessment of activated coagulation time (ACT) during the CTO recanalization procedures. This opinion was more common among operators performing a greater number of procedures. As a target $22 \%$ indicated ACT higher than $200 \mathrm{~s}, 51 \%$ more than $250 \mathrm{~s}$, and $15 \%$ above 300 s; $2 \%$ were not able to indicate any value. Activated coagulation time higher than $300 \mathrm{~s}$ was indicated by operators with longer work experience (Figure 1) and greater experience with CTO.

The need to use over-the-wire (OTW) balloons or microcatheters from the beginning of the CTO recanalization procedure was revealed by $39 \%$ of study participants. This opinion was more frequent among operators performing more than 50 CTOs per year.

Preferred first-choice wires in CTO angioplasty predominantly were Fielder XT (41\%), Pilot (26\%) and Whisper MS (14\%). Also as a first-choice wire BMW (8\%), Sion Blue (7\%), Progress (3\%) and CrossIT (1\%) were men- 
tioned. Operators with longer work experience, with cardiology specialization, performing more CTO procedures per year and with a greater success rate indicated Fielder XT more frequently as a first-choice wire.

As the second-choice wire most often Miracle (38\%), Pilot (21\%) and Fielder XT (13\%) wires were indicated. Some respondents also indicated Progress (8\%), Whisper MS (8\%), CrossIT (5\%), Confianza (3\%), BMW (2\%) and Sion Blue $(2 \%)$ as the second-choice wires. Miracle as a second-choice wire was chosen more frequently by physicians with longer work experience and cardiology specialization, performing more CTO procedures per year, more often using OTW balloons and microcatheters as well as preferring "soft" wires as a first-choice wire.

As the third-choice wire in the CTOs the majority indicated Confianza (47\%), Miracle (19\%) and Pilot (11\%). Third-choice wires for $23 \%$ of respondents were: CrossIT, Terumo, Whisper MS, Fielder XT and Sion Blue. Likewise as in second-choice wires, cardiologists with longer work experience and performing more CTO procedures per year more frequently chose Confianza.

The most frequent sequence of preferred wires was: a soft wire as the first one, then a hard wire dedicated to CTO with gradually increasing tip stiffness, and a hard wire dedicated to CTO with a tapered tip as the last one. That order was chosen by more experienced operators and those who accepted greater radiation doses during recanalization procedures.

Almost half of studied physicians (47\%) agreed that contralateral contrast injection is necessary in over $70 \%$ of procedures. This opinion was supported by operators with longer work experience and those who allow greater radiation doses during recanalization procedures, but was not associated with experience in CTO procedures.

When evaluating the success rate and safety of retrograde technique in comparison to antegrade, one third of the studied group stated that retrograde technique has similar success and complications rates, $48 \%$ thought that retrograde technique has a similar success rate and more frequent complications, and only $19 \%$ of respondents stated that retrograde has a lower success rate and more complications than antegrade technique.

Questions regarding procedural aspects of retrograde technique were answered only by $58 \%$ of physicians. The rest wrote in the comments that they do not use this technique or they are too inexperienced to answer these questions. Twenty-five percent of respondents from the group of physicians using retrograde technique stated that in case of antegrade technique failure after 5-10 min of fluoroscopy they switch to the retrograde technique. The rest of the physicians (75\%) switch to retrograde technique only after utilizing all options of antegrade technique, but still before creation of large artery dissection. Twenty-six percent of respondents use the retrograde strategy for each second approach to CTO recanalization and $71 \%$ use this technique as a method of choice in case of ostial occlusion or stumpless CTOs. Slightly more than half of operators (52\%) use "collateral surfing" technique and $48 \%$ prefer selective contrast injection to collaterals. Most of the respondents prefer switching to retrograde after a failed antegrade approach in the next session, and only $28 \%$ prefer doing so during the same procedure.

According to $16 \%$ of respondents, "subintimal tracking \& reentry" technique (STAR/MiniSTAR) is a very good method which they use very often. Forty-five percent of respondents think it should be used in about a quarter of procedures. Thirty-nine percent state that this technique should be avoided.

The importance of intravascular ultrasound (IVUS) is appreciated by $49 \%$ of physicians in antegrade technique and by $52 \%$ in retrograde technique. If IVUS could be available without any limits, respondents stated that they would have used it in $55 \%$ of procedures (from $10 \%$ to $100 \%$, median $50 \%$ ).

In most cases (93\%) operators limit the amount of contrast based on the value of the glomerular filtration rate (GFR). Most frequently (52\% of answers) the upper limit of contrast was indicated as 4-6 $\times$ GFR ml. In $36 \%$ of cases operators stop the procedure when the volume of contrast reaches $6-8 \times$ GFR $\mathrm{ml}$ and $12 \%$ of physicians accept as high contrast volume as $8 \times \mathrm{GFR} \mathrm{ml}$. Interestingly, the lowest upper limit of contrast (4-6 $\times$ GFR) was indicated more frequently by operators with shorter work experience (Figure 2).

When planning the second attempt of recanalization after the first one failed, $73 \%$ of operators prefer waiting for more than 4 weeks, $21 \%$ think that it can be done during 1-4 weeks after the first attempt, and $6 \%$ wait less than 2 weeks.

The highest dose of radiation accepted by $72 \%$ of respondents is 5 Gy (it was also the lowest radiation dose that could be chosen in the questionnaire), $24 \%$ of oper-

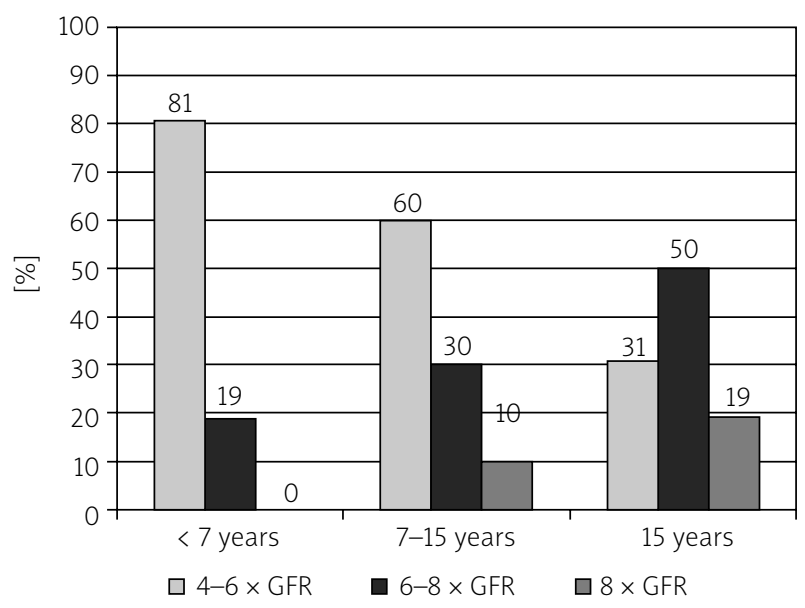

Figure 2. Maximum accepted contrast volume according to the work experience 


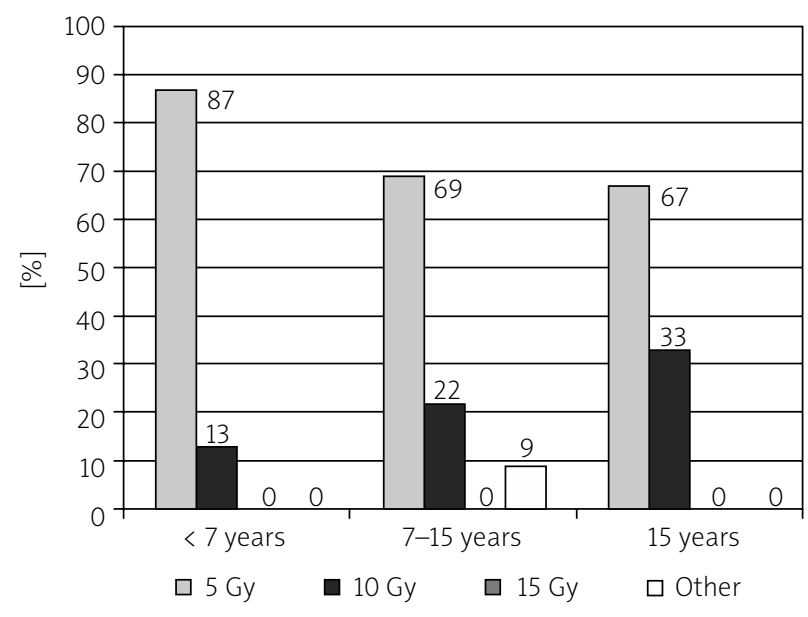

Figure 3. Maximum accepted radiation dose during CTO recanalization according to the work experience

ators accept a radiation dose not greater than $10 \mathrm{~Gy}$, and none chose the highest radiation dose of 15 Gy. Three percent of respondents accepted a different radiation dose, which was not given in the answers. The lowest accepted dose was more often chosen by respondents with shorter work experience, without cardiology specialization, with less experience in СTO procedures and those who accept lower contrast volumes during procedures (Figure 3).

Seventy-one percent of physicians pay attention to the number of frames during fluoroscopy, stating that their preferred rate is 12 frames per second (fps) on average (from 6.25 to $30 \mathrm{fps}$, median 10 ). Only $31 \%$ of operators paying attention to fps preferred 7.5 or fewer fps during the procedure.

\section{Discussion}

The presented study is the first attempt to assess the knowledge about technical aspects of percutaneous CTO recanalization among Polish interventional cardiologists. In our previous study we were able to present the knowledge of this topic among Polish physicians only with an interest in cardiology [4]. In our opinion the survey conducted during two major Polish interventional cardiology workshops ensures representativeness of the study sample. The mean working time of surveyed physicians was 13 years. The majority had passed the cardiology examination, and also the majority worked in departments of cardiology with a catheterization laboratory. More than two-thirds were certified as an independent operator.

Since CTO procedures are typically long and require the use of multiple intracoronary devices, prevention of intracoronary thrombosis and catheter thrombosis are issues of the highest importance [5]. The need for routine assessment of ACT during the CTO recanalization was confirmed by the majority of respondents (86.5\%). Half of respondents indicated a target value of $250 \mathrm{~s}$, which in the common belief is a sufficient one. Only a few physicians chose ACT > $300 \mathrm{~s}$, which is recommended by the EuroCTO Club, especially during retrograde procedures [5, 14, 15].

In the EuroCTO Club consensus published in 2007 it is clearly indicated that the wires dedicated to CTO recanalization should be used in combination with an OTW microcatheter or balloon [15]. This allows exchange of a floppy wire for a dedicated stiffer CTO wire, but also facilitates transmission of torque to the wire tip and improves tactile feedback. Furthermore, it allows adjustment of the wire tip curves throughout the procedure [14].

The technique of $\mathrm{PCl}$ in chronic occlusions has changed dramatically in the last 20 years due to increased operator experience and the development of improved, dedicated equipment, including the development of dedicated guidewires. The latest addition in this category is the Fielder family (Asahi Intecc Co., Nagoya, Japan). These wires confer higher trackability and manoeuvrability in comparison with other polymeric guidewires. The introduction of such wires, especially the Fielder XT wire, has dramatically changed the way in which CTOs are approached. They have become the starting wires of choice since in $40 \%$ of cases they can cross even long occlusions or in case of failure they do not easily create large dissections [5]. The same number of Polish cardiologists (41\%) indicate these wires as the first choice ones.

The CTO procedures are highly individualized, but there are some general principles in the selection and use of wires. We know that in a true CTO which is more than 3 months old it is unlikely that a floppy guide wire will cross the occlusion [14]. Such types of wires are often the best initial choice to negotiate the segment proximal to the occlusion and advance an OTW catheter up to the proximal stump and then change to a stiffer dedicated wire [14]. Despite this, 22\% indicate the Whisper MS and $\mathrm{BMW}$ as the first choice wire. Also those wires were indicated as second-, and third-choice wires.

Less than half of respondents selected stiffer wires such as the Miracle family and Confianza family as second- and third-choice wires.

When the distal vessel is mainly filled by retrograde collaterals, or there are bridging collaterals originating near the occlusion that are likely to have their flow impaired after wire-catheter advancement, contralateral injection is advisable from the start of the procedures, and such injections are used in $62 \%$ of cases done by dedicated CTO operators [5]. In our survey, half of the respondents agreed with the statement that contralateral injection is necessary in more than $70 \%$ of cases.

Since 2005, when Katoh was the pioneer in the field introducing controlled antegrade and retrograde subintimal tracking (CART) technique, it became the next step in improving the CTO recanalization efficacy [16]. The novelties introduced in this procedure were the targeted septal collateral crossing with dedicated wires and microcatheters [17]. The retrograde technique represents 
a breakthrough in CTO recanalization, with success rates exceeding $90 \%$ in complex CTOs, and it has comparable complication rates with contemporary antegrade techniques [16]. In our survey $48 \%$ of physicians indicated higher complication rates and similar efficacy and 19\% lower success rates and higher complication rates reflecting insufficient knowledge of this technique.

The answers concerning procedural aspects of retrograde technique are in the majority in concordance with EuroCTO Club consensus [5]. Current evidence suggests that the retrograde approach should be reserved for a second attempt after antegrade failure or a strategy of choice in very complex CTOs when the expected antegrade success rate is below $50 \%$ [5]. It must be emphasized that this technique should be reserved for very experienced antegrade operators (experience of more than 300 CTO cases, and $>50$ per year). A minimum of 50 retrograde procedures (25 as second operator and 25 as first under supervision) are required before a cardiologist becomes an independent retrograde operator [5].

Only $39 \%$ of respondents agree with the statement of the EuroCTO Club that subintimal tracking techniques should be applied only as a bail-out [5].

The IVUS can be applied during the procedures of CTO recanalization for different purposes: 1) the identification of the occlusion site in stumpless (TO; 2) to document a subintimal location of the wire and to facilitate the re-entry of a second wire in the true lumen (IVUS-guided re-entry); 3) during retrograde CTO recanalization to monitor the retrograde wire course, to properly size the balloon during CART and reverse-CART, and to avoid the risk of extending dissections caused by antegrade contrast injections; 4) after wire crossing to measure the vessel size and length and optimize stent apposition and expansion $[5,18]$. In Europe IVUS is used only in $2 \%$ of CTO procedures, reflecting the necessity for 7 or 8 Fr guiding catheters (the latter not routinely used in Europe), the additional skills in image interpretation, and different reimbursement policies $[5,19]$. This is also reflected in the opinion of respondents of our survey, that they would like to use IVUS in more than $50 \%$ of procedures, but we must state that routine use of IVUS is not recommended as a routine procedural imaging tool for CTO PCI in the absence of randomized efficacy data [5].

Of paramount significance is to prevent contrast-induced nephropathy (CIN) during the CTO recanalization procedure. Most operators would wish to keep dye load even in patients with normal eGFR less than $400 \mathrm{ml}$; however, some have suggested that up to $500-600 \mathrm{ml}$ can be tolerated [5], although in the EuroCTO Club consensus the maximal amount is defined as $4 \times \mathrm{GFR}(\mathrm{ml})$ $[5,20]$. Half of the operators are in concordance with this statement.

When planning the second attempt, three quarters of respondents prefer waiting 4 weeks, and this is consistent with 3-4 weeks advised in the consensus [5].
Exposure to radiation is an important consideration, since it is prolonged during CTO cases as compared to $\mathrm{PCI}$ for non-occlusive lesions. During PCI of CTOs the physician should be aware that he needs to make every effort to reduce radiation exposure and to document radiation exposure during the procedure [5]. There are several methods to reduce the radiation and optimize radiation exposure for both operator and patient [5]. One of these is the pulse and frame rate for fluoroscopy and imaging reduced to 7.5 pulses/s instead of higher rates providing sufficiently high quality. But those numbers were indicated by one third of respondents. The procedure should be stopped when radiation reaches a maximum of $10 \mathrm{~Gy}$, and the operator should be alerted when radiation reaches 5 Gy [5]. Our respondents were more cautious; in the opinion of $73 \%$ the maximal dose is 5 Gy and only $24 \%$ accept the maximal dose of $10 \mathrm{~Gy}$.

\section{Conclusions}

The awareness of procedural aspects (particularly the retrograde technique) as well as the dedicated equipment (wires, microcatheters and other CTO dedicated devices) is still insufficient. An independent primary operator for most angioplasty procedures does not automatically translate into an ability to approach any CTO. Continuous dedicated CTO training and a minimal annual number of procedures to maintain and further develop the skills are crucial in achieving satisfactory success rates with low complication risk.

\section{Conflict of interest}

The authors declare no conflict of interest.

\section{References}

1. Di Mario C, Werner GS, Sianos G, et al. European perspective in the recanalisation of Chronic Total Occlusions (CTO): consensus document from the EuroCTO Club. Eurointervention 2007; 3: $30-43$

2. Hoebers LP, Claessen BE, Dangas GD, et al. Contemporary oveview and clinical perspectives of chronic total occlusions. Nat Rev Cardiol 2014; 11: 458-69.

3. Fefer P, Knudtson ML, Cheema AN, et al. Current perspectives on coronary chronic total occlusions: the Canadian Multicenter Chronic Total Occlusions Registry. J Am Coll Cardiol 2012; 59: 991-7.

4. Bryniarski L, Zabojszcz M, Bryniarski K, Terlecki M. Knowledge about chronic total coronary artery occlusions among Polish physicians. Postep Kardiol Inter 2010; 6: 66-70.

5. Sianos G, Werner GS, Galassi AR, et al. Recanalisation of chronic total coronary occlusions: 2012 consensus document from the EuroCTO Club. Eurointervention 2012; 15: 139-45.

6. Werner GS, Figulla HR. Direct assessment of coronary steal and associated changes of collateral hemodynamics in chronic total coronary occlusions. Circulation 2002; 106: 435-40.

7. Werner GS, Fritzenwanger M, Prochnau D, et al. Determinants of coronary steal in chronic total coronary occlusions donor artery, collateral, and microvascular resistance. J Am Coll Cardiol 2006; 48: $51-8$ 
8. Syrseloudis D, Secco GG, Barrero A, et al. Increase in J-CTO lesion complexity score explains the disparity between recanalisation successand evolution of chronic total occlusion strategies: in sights from a single-center 10-year experience. Heart 2013; 99: 474-9.

9. Bryniarski L, Surowiec S, Klima $Ł$, et al. Recanalization of coronary chronic total occlusion by retrograde approaches - the first experience in Poland. Kardiol Pol 2015; 73: 167-76.

10. Patel VG, Brayton KM, Tamayo A, et al. Angiographic success and procedural complications in patients undergoing percutaneous coronary chronic total occlusion interventions: a weighted meta-analysis of 18,061 patients from 65 studies. J Am Coll Cardiol Interv 2013; 6: 128-36.

11. Drozd J, Wójcik J, Opalińska E, et al. Percutaneous angioplasty of chronically occluded coronary arteries: long-term clinical follow-up. Kardiol Pol 2006; 64: 667-73.

12. Brilakis ES, Karmpaliotis D, Werner GS, et al. Developments in coronary chronic total occlusion pecutaneous coronary interventions: 2014 state-of-the-art update. J Invasive Cardiol 2014; 26: 261-6.

13. Bryniarski KL, Zabojsz M, Dębski G, et al. What Polish interventional cardiologists know about indications and qualification for recanalisation of chronic total coronary artery occlusions? Kardiol Pol 2015, ahead of print, DOI: 10.5603/KP.a2015.0091.

14. Di Mario C, Werner GS, Sianos G, et al. European perspective in the recanalization of Chronic Total Occlusions (CTO): consensus document from the EuroCTO Club. Eurointervention 2007; 3: $30-43$.

15. Sianos G, Varlis P, Di Mario C, et al. European experience with the retrograde approach for the recanalization of coronary artery chronic total occlusions. A report on behalf of the EuroCTO Club. Eurointervention 2008; 4: 84-92.

16. Surmely JF, Tsuchikane E, Katoh O, et al. New concept for CTO recanalization using controlled antegrade and retrograde subintimal tracking: the CART technique. J Invasive Cardiol 2006; 18: 334-8.

17. Tsuchikane E, Katoh O, Kimura M, et al. The first clinical experience with a novel catheter for collateral channel tracking in retrograde approach for chronic coronary total occlusions. JACC Cardiovasc Interv 2010; 3: 165-71.

18. Tsujita K, Maehara A, Mintz G, et al. Intravascular ultrasound comparison of the retrograde versus antegrade approach to percutaneous intervention for chronic total coronary occlusions. JACC Cardiovasc Interv 2009; 2: 846-54.

19. Galassi AR, Tomasello SD, Reifart N, et al. In-hospital outcomes of percutaneous coronary intervention in patients with chronic total occlusions: insights from the ERCTO (European Registry of Chronic Total Occlusion) registry. Eurointervention 2011; 7: 472-9.

20. Laskey WK, Jenkins C, Selzer F, et al. Volume-to-creatinine clearence ratio: a pharmacokinetically based risk factor for prediction of early creatinine increase after percutaneous coronary intervention. J Am Coll Cardiol 2007; 50: 584-90. 